\title{
Occupationally Related Outbreak of Chickenpox in an Intensive Care Unit
}

\author{
Nasser Yehia A. Aly a,b Ina'am Al Obaid ${ }^{c} \quad$ Noura Al-Qulooshid ${ }^{d}$ Zahida Zahed $^{d}$ \\ ${ }^{a}$ Department of Tropical Medicine and Hygiene, Faculty of Medicine, University of Alexandria, Alexandria, Egypt; \\ ${ }^{b}$ Directorate of Infection Control, Departments of ${ }^{\mathrm{C}}$ Laboratories and ${ }^{\mathrm{d}}$ Infection Control, Al-Sabah Hospital, \\ Ministry of Health, Kuwait
}

\section{Key Words}

Varicella zoster virus · Chickenpox outbreak •

Intensive care unit

\begin{abstract}
Objective: To report occupationally related outbreak of chickenpox in intensive care unit (ICU). Case Presentation and Intervention: The index patient was a 4-year-old child who presented with a 3-day history of fever and rash and was clinically diagnosed as chickenpox encephalitis. She was admitted to an isolation room in ICU, kept on oxygen mask and given intravenous fluids, anticonvulsant, antipyretic and acyclovir. Twelve hours later, the patient was transferred to Infectious Diseases Hospital. Secondary cases were three unvaccinated ICU staff nurses who developed chickenpox 1621 days following exposure. They were also transferred to Infectious Diseases Hospital. The affected nurses were interviewed and filled out a questionnaire. Individual immune status was verified by reviewing previous varicella zosterIgG screening data for all ICU staff. The chickenpox case was defined according to the CDC case classification. All were treated with no complications. Conclusion: This report shows that adherence to isolation precautions, exclusion of susceptible staff from attending the affected patient, education, pre-employment anti-VZV-lgG screening and vaccine coverage of staff could have prevented the occurrence of this outbreak.

Copyright $\odot 2007$ S. Karger AG, Basel
\end{abstract}

\section{Introduction}

Chickenpox is a highly contagious and common disease of childhood caused by varicella zoster virus (VZV) and associated with viremia and a diffuse rash $[1,2]$. Transmission of infection is primarily by direct contact with infectious virus in skin lesions or by air-borne transmission of respiratory secretions [3]. What makes the introduction of the virus in hospital environment so special is that it can affect individuals at high risk of varicella-associated complications. Consequently, hospitals make strenuous efforts to prevent spread of VZV from infected staff and patients to susceptible persons [4]. Despite such efforts, nosocomial transmission still occurs and poses a substantial risk to both patients and healthcare workers. The objective of this report was to highlight an outbreak of chickenpox among susceptible staff in an intensive care unit (ICU) setting.

\section{Case Report}

The index case was a 4-year-old female who presented to the Pediatric Casualty with a 3-day history of fever and rash. On examination, she was unconscious and had generalized tonic-clonic convulsions. She had a characteristic diffuse rash in the form of blisters on a red base mainly on the face, neck and trunk. Her temperature was $38.5^{\circ} \mathrm{C}$. Chest and abdominal examinations were normal except for the rash. Laboratory investigation gave

\section{KARGER}

Fax +4161306 1234

E-Mail karger@karger.ch

www.karger.com
(C) 2007 S. Karger AG, Basel

$1011-7571 / 07 / 0165-0399 \$ 23.50 / 0$

Accessible online at:

www.karger.com/mpp
Nasser Yehia A. Aly

Directorate of Infection Control, Ministry of Health

PO Box 12414

Al-Shamiya 71655 (Kuwait)

Tel. +965 488 8000, ext. 3816, Fax +965 488 2684, E-Mail nasseryehia@gmail.com 
Table 1. History and clinical findings of the affected nurses

\begin{tabular}{llll}
\hline Variable & N1 & N2 & N3 \\
\hline Age/gender & $26 / \mathrm{F}$ & $26 / \mathrm{F}$ & $32 / \mathrm{F}$ \\
Nationality & Indian & Indian & Indian \\
Pregnancy & no & no & no \\
Past history of similar illness & no & no & no \\
Previous exposure to a chickenpox case & no & no & yes \\
Immune status & unknown & negative & negative \\
Previous vaccination against chickenpox & no & no & no \\
Nurse was aware of the patient diagnosis & yes & yes & yes \\
Wearing N95 mask & no & no & no \\
Routine nursing in the patient room & yes & yes & no \\
Direct contact with the patient & yes & yes & no \\
Time assigned for patient care in the ICU, h & 8 & 4 & 0 \\
Time spent in the patient room before patient & & & \\
$\quad$ discharge, $h$ & 3 & 1 & 0 \\
Entering patient room after discharge & no & yes & yes \\
Time spent in patient room after discharge, h & 0 & 1 & 0.5 \\
Post-exposure VZIG given & no & no & no \\
Incubation period, days & 16 & 20 & 21 \\
Fever & yes, high & yes & yes, mild \\
Headache & yes & yes & yes, mild \\
Sore throat & yes & yes & no \\
Myalgia and body aches & yes, severe & yes & yes \\
Fatigue & yes & no & yes \\
Rash $>50$ in number & yes & yes & yes \\
Complications (e.g. pneumonitis) & no & no & no \\
Work absenteeism, days & 15 & 14 & 10 \\
\hline
\end{tabular}

hemoglobin: $12.3 \mathrm{~g} / \mathrm{l}$; RBCs: $4.57 \times 10^{12} / \mathrm{l}$; WBCs: $6.7 \times 10^{9} / 1$ with $50.6 \%$ lymphocytes and $15.2 \%$ monocytes; platelets: $133 \times$ 10 $/$; blood glucose: $5.3 \mathrm{mM}$; BUN: $3.7 \mathrm{mM}$; creatinine: $41 \mathrm{mM}$; sodium: $132 \mathrm{~mm}$, and potassium: $3.7 \mathrm{~mm}$. Chest X-ray was normal. The child was diagnosed as chickenpox encephalitis on clinical ground by the pediatrician. She received mannitol 10\%, antipyretic, and anticonvulsant in the casualty. She was then admitted to an isolation room in the ICU and kept on $\mathrm{O}_{2}$ mask. Intravenous fluids, anticonvulsant, antipyretic and acyclovir were given. Next morning, she became fully conscious, afebrile with no neurological deficits and was put on oral diet. After $12 \mathrm{~h}$, she was transferred to Infectious Diseases (ID) Hospital.

Secondary cases were 3 ICU staff nurses (N1, N2 and N3) who developed the illness 16-21 days later. They were also referred to ID Hospital for management. The nurses were excluded from work until they became no longer infectious according to infection control protocols. They were given sick leave (range, 10-15 days) by the ID doctor until the entire rash crusted and they became well. The history and clinical findings of the 3 nurses are summarized in table 1 . Systematic chart review of the index patient was done. The affected nurses were interviewed and their general condition and skin lesions were further assessed. They filled out a questionnaire separately. Individual immune status was verified by reviewing previous VZV-IgG screening data of all ICU staff. The chickenpox case was defined according to the CDC case classification [5].

\section{Discussion}

Four parameters could be used to discuss this outbreak: the setting (ICU), the infectious routes, the incubation period, and the secondary cases (nurses).

\section{Outbreak Setting}

The setting was a 13-bed medical-surgical ICU which includes two isolation rooms. The ventilation system ensures $100 \%$ fresh air with about 20 air changes per hour. The isolation rooms have separate exhaust ventilation. A review of ventilation system was initiated to determine the need for additional air-cleaning methods like mobile HEPA filters in isolation rooms.

\section{Infection Routes}

Two nurses, N1 and N2 (table 1), were most probably directly infected while attending to the patient with chickenpox when they were checking vital signs, giving medication and performing back care. N3 was not assigned with care of the patient. The infection could have been transmitted by air since N3 entered the room after 
the discharge of the patient and before terminal cleaning and disinfection of the room. She helped N2 to clean the mattress cover and bed rails.

\section{Incubation Period}

It is known that the characteristic rash of chicken pox develops after an incubation period of 10 to 21 days [6]. VZV is transmissible for $24-48 \mathrm{~h}$ before rash onset, or 4-5 days after the rash appears [1]. The rash appeared on the nurses 16-21 days after patient admission that is within the known range of incubation period. The possibility that the source of infection to $\mathrm{N} 3$ could be $\mathrm{N} 1$ and $\mathrm{N} 2$ was excluded because $\mathrm{N} 1$ and $\mathrm{N} 2$ developed rash 6 days and 1 day, respectively, before N3. Therefore, the incubation period would be less than the known accepted period. There was no history to suggest extra-hospital exposure to any of the nurses.

\section{Secondary Cases (Nurses)}

N1 was newly employed and her immunity status had not yet been determined. N2 and N3 were identified as seronegative during a previous screening program. They were aware of their immunity status. Despite this, N2 was assigned for patient care. Other members of staff were found immune. Nurses were not vaccinated against VZV and none wore N95 respirator. The nurses also waived exposure reporting.

\section{Conclusion}

This report shows that adherence to isolation precautions, exclusion of susceptible staff from attending the patient, education, pre-employment anti-VZV-IgG screening and vaccine coverage of staff could have prevented the occurrence of this outbreak.

\section{References}

1 Grose C: Variation on a theme by Fenner: the pathogenesis of chickenpox. Pediatrics 1981; 68:735-737.

2 CDC: Varicella; in Atkinson W (ed): Epidemiology and Prevention of Vaccine-Preventable Diseases. Atlanta, Centers for Disease Control and Prevention 2005, pp 159-175.
3 Sawyer MH, Wu YN, Chamberlin CJ, Burgos C, Brodine SK, Bowler WA, La Rocco A, Oldfield EC 3rd, Wallace MR: Detection of varicella-zoster virus DNA in the oropharynx and blood of patients with varicella. J Infect Dis $1992 ; 166: 885-888$.

4 Wreghitt TG, Whipp J, Redpath C, Hollingworth W: An analysis of infection control of varicella-zoster virus infections in Addenbrooke's Hospital Cambridge over a 5-year period 1987-92. Epidemiol Infect 1996;117: 165-171.
5 Jumaan A, Hughes H, Schmid S, Galil K, Plott K, Zimmerman L, Seward J: Varicella; in Wharton M, Hughes H, Reilly M (eds): Manual for the Surveillance of Vaccine-Preventable Diseases. Atlanta, 2002, pp 11-21.

6 Arvin AM: Varicella-zoster virus; in Knipe DM, Howley P (eds): Fields Virology, ed 4. Philadelphia, Lippincott Williams \& Wilkins, 2001, pp 2731-2768. 